\title{
Determination of nutrient uptake kinetic parameters: a comparison of methods
}

\author{
P. J. Harrison ${ }^{1}$, J. S. Parslow ${ }^{2}$, H. L. Conway ${ }^{3}$ \\ ${ }^{1}$ Depts of Oceanography and Botany, The University of British Columbia, Vancouver, British Columbia, Canada V6T 2B1 \\ ${ }^{2}$ School of Australian Environmental Studies, Griffith University, Nathan, Brisbane, Queensland 4111, Australia \\ ${ }^{3}$ Freshwater Research Division, Brookhaven National Laboratory, Upton, New York 11973, USA
}

\begin{abstract}
Three methods which have been used to determine nutrient uptake kinetic parameters were compared using steady-state $\mathrm{NH}_{4}$-limited cultures of the chrysophyte Pseudopedinella pyritormis. The first 2 methods involved a multiple flask incubation where different concentrations of substrate were added to each flask. Method 1 used a variable incubation time, while the incubation time of Method 2 was short and constant. The third method, the perturbation method, involved one large addition of the substrate to one culture and hence the nutritional past history varied throughout the experiment. This method was used also with the diatoms Skeletonema costatum and Chaetoceros debilis in Si-limited chemostats. Results indicate that, for nutrient-limited cultures, kinetic parameters are best estimated using multiple additions of the substrate and a short constant incubation time (Method 2). It appears that this method determines membrane transport, which is still not completely free of feedback inhibition even when the incubation time is very short (e.g. $2 \mathrm{~min}$ ). The short incubation time is necessary because the maximal uptake velocity $\left(V_{\mathrm{m}}\right)$ decreases with increasing incubation time especially for phosphate and ammonium. Method 3 provides valuable information on a third parameter, $V_{i}$, the approximate assimilation rate of the limiting nutrient, that is not obtained by the other methods. Multiple sequential additions of the limiting nutrient to $\mathrm{N}$ - or Si-limited Skeletonema costatum and Chaetoceros debilis revealed that if the additions were small (e.g. $2 \mu M \mathrm{NH}_{4}$ ), there was no change in subsequently determined nutrient uptake kinetic parameters. If the sequential additions were larger (e.g. $6 \mu M$ ) then the maximal uptake rate slowed with time
\end{abstract}

\section{INTRODUCTION}

When one nutrient becomes limiting in the aquatic environment, the uptake rate of this limiting nutrient can be described by a rectangular hyperbola, similar to the Michaelis-Menten equation for enzyme kinetics, $V=V_{\mathrm{m}} \cdot S /\left(K_{S}+S\right)$ where $V$ is the uptake velocity $\left(\mathrm{h}^{-1}\right), V_{\mathrm{m}}$ the maximal uptake velocity, $S$ the concentration of limiting nutrient and $K_{S}$ the half-saturation constant representing the value of $S$ where $V=V_{\mathrm{m}} / 2$. The species-specific nutrient uptake kinetic parameters, $V_{\mathrm{m}}$ and $K_{\mathrm{S}}$ may be used to explain species competition involving a limiting nutrient (Dugdale 1967, Tilman 1977, Button 1985). Since these early studies it has been realized that $V_{\mathrm{m}}$ is frequently variable over time and that a time course incubation must be run in order to determine the complete pattern of response of nutrient-limited cells to an addition (pulse) of the limiting nutrient. Consequently, the 2 parameters $V_{\mathrm{m}}$ and
$K_{S}$ do not fully describe an organism's response to a nutrient pulse and more parameters are needed.

Nutrient uptake is generally determined by measuring the decreasing concentration of the limiting nutrient in the culture medium or by measuring the incorporation of isotopes such as ${ }^{15} \mathrm{~N}$ (Dugdale \& Goering 1967), ${ }^{32} \mathrm{P}$ (Cembella et al. 1984), and ${ }^{30} \mathrm{Si}$ (Nelson \& Goering 1977) to determine nutrient incorporation into the cell.

There are 3 general categories of methods for measuring nutrient disappearance from the medium and hence nutrient uptake rates (Fig. 1). There are many variations in the first type of method which was frequently used in the early days to determine nutrient uptake kinetics. Often a series of 5 to 10 flasks were set up and a different concentration of the limiting nutrient was added to each flask (e.g. Eppley \& Thomas 1969, Eppley et al. 1969). The experiment was often initiated by adding a subsample (e.g. 100 to $200 \mathrm{ml}$ ) from a batch 


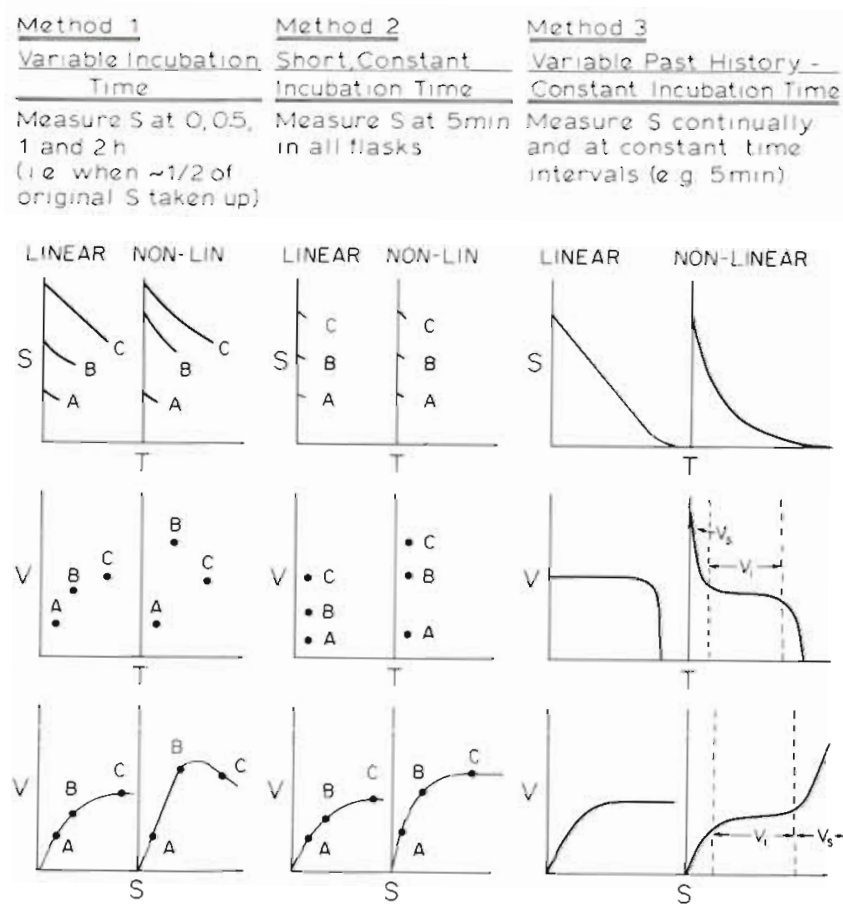

Fig. 1. Determination of uptake rates by the disappearance of the limiting nutrient from the culture medium using 3 different methods. Hypothetical curves for the disappearance of a limiting nutrient such as ammonium with time ( $S$ vs $T$ ) and the associated $V$ vs $T$ and $V$ vs $V$ curves are given for each method. For each method, graphs describing uptake of a nutrient by nutrient-saturated cells which exhibit linear disappearance (LINEAR) of the nutrient from the medium and uptake of a nutrient by nutrient-limited cells which exhibit non-linear (NON-LINEAR) disappearance of the limiting nutrient from the culture medium are given. Symbols are defined in the text

culture that had just run out of nutrients or a nutrientlimited chemostat culture to each of the flasks. These flasks were incubated for different times (often the time when the limiting substrate concentration was reduced to approximately half the concentration of the original addition). The uptake rate for the incubation period was plotted as a function of either the initial or the mean substrate concentration in the flasks. Therefore, in this type of method, the cellular past history varied because the incubation time varied and the initial substrate concentration added to each flask also varied.

The second approach is identical to the first, except, that the incubation time for each flask is the same and relatively short. Incubation times of a few minutes have been used in an attempt to alleviate recent past history effects, perhaps from feedback inhibition, during the uptake experiments (McCarthy \& Goldman 1979, Goldman \& Glibert 1982, Quarmby et al. 1982).

Another approach to measuring nutrient uptake rates was developed by Caperon \& Meyer (1972), and termed the perturbation experiment. The experimental culture was grown in a chemostat and upon reaching steady state, the culture was perturbed by adding a relatively large spike addition of the limiting nutrient (e.g 5 to $10 \mu M$ ) directly to the chemostat. Continual sampling and analyses with an AutoAnalyzer ${ }^{\circledR}$ provided a time series of disappearance of the limiting nutrient from the culture until steady state was regained or the limiting nutrient was completely taken up. This time series approach was also used by Conway et al. (1976) and others with some variations in the exact detail of the methods and data analysis. In this type of method the time of incubation is also constant and it is often calculated as time interval between successive samples. However, the past history is variable because the cells at the end of the experiment ( 2 to $12 \mathrm{~h}$ ) have been exposed to relatively high levels of substrate for a longer period of the time than those at the beginning of the experiment.

Since it is unclear how the choice of methods affects the values of $V_{\mathrm{m}}$ that are determined, a systematic comparison of these 3 methods was undertaken in this study. The time course of nutrient disappearance for different nutrients was examined, and new parameters are suggested to fully describe the response in uptake rate of a limiting nutrient.

\section{TERMINOLOGY AND CONCEPTS}

Uptake of a nutrient addition by phytoplankton appears to follow Michaelis-Menten kinetics for certain species and/or under certain growth conditions. In such cases the $V$ vs $S$ plot is hyperbolic and the reciprocal plot (e.g. $S / V$ vs $S$ ) is linear. In this case $V_{m}$ is independent of time (i.e. it is constant over time and has only one value, as long as $S$ is saturating). In other cases, $V_{\mathrm{m}}$ changes during the course of uptake of the nutrient spike and the $V$ vs $S$ plot is not hyperbolic and the reciprocal plot is non-linear (i.e. at the highest $S$ values the data points fall below the line because of the very high initial $V$ values). The best documented case of non-linearity in such plots is a decrease in $V_{\mathrm{m}}$ within a few minutes or even tens of seconds after the nutrient spike. In this case $V_{\mathrm{m}}$ is time-dependent and these changes in the value of $V_{\mathrm{m}}$ with time have been described by 2 sets of symbols. The first set denotes a precise time interval (in min) over which an average value of $V_{\mathrm{m}}$ is measured as a superscript (e.g. $V_{\mathrm{m}}^{0-1}$, $V_{\mathrm{m}}^{1-5}, V_{\mathrm{m}}^{5-10}$, etc.) (Goldman \& Glibert 1983). The second set divides the maximal uptake period into 2 parts, the initial rapid uptake, $V_{\mathrm{s}}$ (surge uptake), and the slow decline or almost constant uptake rate with time, $V_{\mathrm{i}}$, (internally controlled uptake) (Conway et al. 1976). The symbol $V_{\mathrm{s}}$ represents a maximal uptake rate that declines as an internal pool is filled due to feedback 
inhibition. The period during which $V_{\mathrm{s}}$ is maintained is variable and depends on how long it takes to fill the internal pool. Therefore $V_{\mathrm{s}}$ is really $V_{\mathrm{m}}$ without any superscript time interval designation. $V_{1}$ represents a maximal uptake rate when the internal pool is full and the cell quota is increasing slowly towards a maximal value of the amount of the limiting nutrient per cell. Therefore, $V_{\mathrm{i}}$ is just a value $V_{\mathrm{m}}^{T_{1}-T_{2}}$ where $T_{1}$ and $T_{2}$ are large (i.e. minutes to hours); for example, $V_{\mathrm{i}}=V_{\mathrm{m}}^{5-300}$, representing an average uptake rate over a time interval of 5 min to $5 \mathrm{~h}$. It may not have a constant value because it may continue to slowly decline (if excess nutrients are present) over hours to days until a maximal cell quota is obtained (i.e. one generation time or more).

Of course, over much longer periods of time than discussed above, a nutrient-saturated culture will not exhibit linear nutrient disappearance because, due to exponential cell growth and biomass increase, the rate of nutrient uptake $\left(\mu \mathrm{mol} \mathrm{l}^{-1} \mathrm{~h}^{-1}\right)$ will increase with time but the specific uptake rate $\left(V ; \mathrm{h}^{-1}\right)$ will remain constant until the culture begins to run out of nutrients.

\section{MATERIALS AND METHODS}

Chemostat system and analyses. The chemostat system and source of inoculum of Skeletonema costatum (Grev.) Cleve and Chaetoceros debilis Cleve have been described previously (Davis et al. 1973, Harrison et al. 1977). Pseudopedinella pyriformis N. Carter (NEPCC No. 49) was obtained from the Northeast Pacific Culture Collection, Department of Oceanography, University of British Columbia, Vancouver, Canada. $P$. pyriformis was used in the experiments to compare the 3 methods of measuring uptake rates, while $S$. costatum and $C$, debilis were used in multiple nutrient addition experiments. The cultures were grown in 61 borosilicate flat-bottomed boiling flasks under continuous light with an irradiance of $125 \mu \mathrm{mol}$ quanta $\mathrm{m}^{-2} \mathrm{~s}^{-1}$ as previously described (Harrison et al. 1977). The dilution rate and temperature were $0.5 \mathrm{~d}^{-1}$ and $15^{\circ} \mathrm{C}$ respectively for $S$. costatum and $C$. debilis; for $P$. pyriformis they were $0.8 \mathrm{~d}^{-1}$ and $18{ }^{\circ} \mathrm{C}$.

The methods for nutrient analyses, cell counts and fluorescence were described previously (Davis et al. 1973). Nutrients, trace metals and vitamins were added to artificial seawater to give a final concentration of $f / 50$ except for the limiting nutrient, ammonium or silicate (Guillard \& Ryther 1962). The concentration in the $\mathrm{NH}_{4}^{+}$-limited inflow medium was $0.5,7.8,45$ and $5.5 \mu \mathrm{M}$ of nitrate, ammonium, silicate and phosphate, respectively. The concentrations of nutrients in the Si-limited inflow medium were $10.5,55.0$, and $5.5 \mu \mathrm{M}$ silicate, nitrate and phosphate, respectively. When no trend was observed in the effluent nutrient concentrations, cell numbers or fluorescence for several days, the culture was assumed to be at steady state and the following experiments were initiated.

Since a large $6 \mathrm{I}$ chemostat was used, $200 \mathrm{ml}$ could be removed from the chemostat without appreciably changing the dilution rate $(3.3 \%$ change) and the 200 ml subsample that was removed was replaced by the time the next experiment was performed 1 to $2 \mathrm{~h}$ later (for Methods 1 and 2 ; see below). A small amount of the limiting nutrient was added to the subsample and the culture was immediately incubated under previous growth conditions. Nutrient samples were taken every 2 or 6 min either manually or with an automatic timing device for at least 30 min. Nutrient analyses were made on an AutoAnalyzer ${ }^{(*)}$ and cells were removed before they entered the AutoAnalyzer with an in-line filter. Cells which accumulated on the filter were prevented from taking up nutrients by inactivating them with a $5 \mathrm{ml}$ distilled water wash every $6 \mathrm{~min}$. Near the end of a perturbation experiment (Method 3) the cells frequently released the contents of their internal pools. If nutrient concentrations were determined over a $5 \mathrm{~min}$ sample time, this initial elevated spike in the nutrient concentration could be eliminated as the remainder of the AutoAnalyzer ${ }^{B}$ peak plateaued, representing the true sample concentration. After each subsample was run the flask was rinsed and filled with another $200 \mathrm{ml}$ subsample from the chemostat to which a higher concentration of the limiting nutrient was added and the same continuous sampling was repeated. These series of uptake experiments were performed over a limiting substrate concentration range from 0.5 to $18 \mu M$ for Methods 1 and 2 . The same substrate additions were made to filtered effluent and then measurements of the concentration of the limiting nutrient were made and taken to represent true initial concentrations at $T=0$. Uptake rates were also calculated by taking the initial substrate concentration $2 \mathrm{~min}$ after the actual substrate addition in order to demonstrate the importance of the first $2 \mathrm{~min}$ period.

Method 1 - Variable incubation time, constant past history, and variable substrate addition. Uptake rates were determined as described above. A series of triplicate flasks were set up, filled with $60 \mathrm{ml}$ of culture and spiked to different nutrient concentrations at $2 \mathrm{~min}$ time intervals to accommodate nutrient analysis. Incubation time was variable because it was the time taken for half of the added substrate to disappear from the medium. Uptake rates were calculated and related to the limiting substrate concentration at the middle of the time interval.

Method 2 - Constant incubation time, constant past history and variable substrate addition. A series of triplicate flasks were set up, filled with $60 \mathrm{ml}$ of culture 
and spiked to different concentrations of the limiting nutrient. Constant incubation times (e.g. 2, 6, 18 or 30 min) were used.

Method 3 - Constant incubation time and variable past history (perturbation). After the series of experiments described above, the chemostat was still at the original volume of $6 \mathrm{l}$. At this time the culture was quickly divided into two 31 flasks. One culture was reconnected to the pump, but at half the original flow rate since the chemostat volume had been reduced by half. The other 31 culture was used for the perturbation experiment (Conway et al. 1976). Approximately $6 \mu \mathrm{M}$ of the limiting nutrient was added to the chemostat culture and the disappearance of the nutrient from the medium was followed for the next 4 to 8 h by taking point samples every 5 min until the nutrient was exhausted. If at this time enough of the culture remained a second or third addition of the limiting nutrient was made in order to determine the effect of a recent change in past history on the rate of nutrient uptake.

On some occasions the 3 I culture, which had been kept at steady state while the experiment described above was performed $(>8 \mathrm{~h})$, was used to determine what effect a smaller, but repeated substrate addition would have on the values of $V_{\mathrm{m}}$. The pump was stopped and a small amount $(\sim 2 \mu M)$ was added to the culture and the sampling procedure repeated. From 4 to 7 additions were made in the various experiments with the different species and type of nutrient limitation. In another experiment, 2 or 3 larger additions $(\sim 6$ $\mu M$ ) were added sequentially after the nutrient from each addition was depleted. As soon as nutrient depletion was diagnosed by the AutoAnalyzer ${ }^{(}$(e.g. after 20 min for ammonium analyses) another addition was immediately made to the culture.

Uptake rate calculations. Uptake rates were calculated as described by Conway et al. (1976). The particulate values of nitrogen and silicon used in the calculations were determined at steady state from a mass balance where the disappearance of the limiting nutrient was assumed to be equal to the increase in particulate nitrogen or silicon in the culture. The nutrient uptake kinetic parameters were determined by a direct hyperbolic fit and statistical analysis was made using the program of Cleland (1967).

\section{RESULTS AND DISCUSSION}

\section{Non-linear disappearance of limiting nutrient}

The disappearance of ammonium from the medium was not linear with time and, consequently, the maximal uptake rate of ammonium decreased with increas-

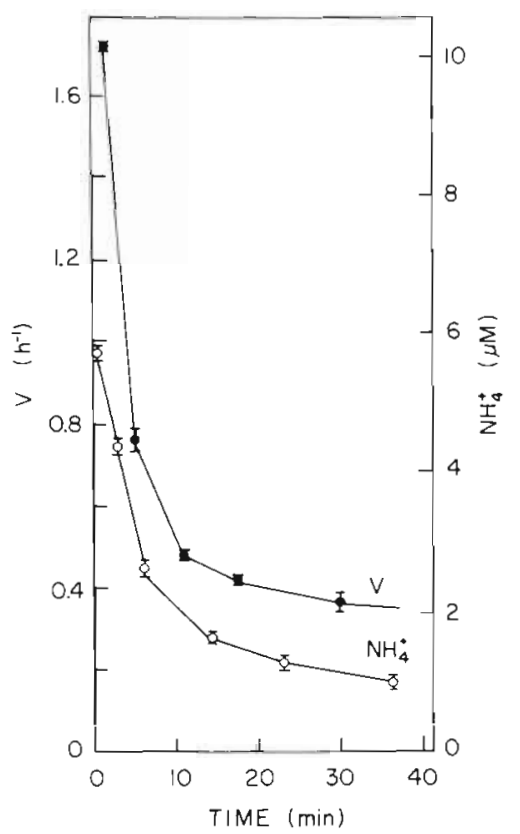

Fig. 2. Change in ammonium concentration (0) over time when an $\mathrm{NH}_{4}^{+}$-limited culture of Pseudopedinella pyriformis was perturbed with $\sigma \mu M$ ammonium at $T=0$. Error bars represent $\pm 1 \mathrm{SD}, n=3$

ing incubation time (Fig. 2). For this reason previous workers have put the time interval over which the measurement of $V_{m}$ was made as a superscript on the $V_{\mathrm{m}}$ term (Goldman \& Glibert 1983, Parslow et al. 1984a).

There were early reports of increased nutrient uptake rate as a result of nitrogen deficiency (Syrett 1956). Eppley et al. (1969) reported non-linear nutrient disappearance and to eliminate this effect on their uptake rate measurements, they preincubated the cells with the limiting nutrient for $1 \mathrm{~h}$ before beginning their uptake rate measurements. Non-linear disappearance of the limiting nutrient from the medium can also be seen in Caperon \& Meyer's data (1972; their Fig. 3) but their fit of a line to the data ignored the rapid uptake of ammonium that occurred in the first $30 \mathrm{~min}$. The importance of rapid uptake of the limiting nutrient was first recognized by Conway et al. (1976) and extended later by McCarthy \& Goldman (1979), Goldman et al. (1981), Goldman \& Glibert (1982), and Parslow et al. (1984b, c). Rapid or enhanced nutrient uptake has now been observed for phosphate, ammonium, silicate and nitrate (in order of decreasing enhancement). Phosphate and ammonium uptake may be enhanced 5 to 50 times over the maximum growth rate (Parslow et al. 1984c). On the other hand, nitrate and silicate uptake is enhanced only if the cells are Si- or N-limited and even then by less than a factor of 2 (Conway et al. 1976 , Collos 1983). If the cells are $\mathrm{Si}$ - or N-starved for $>24 \mathrm{~h}$ 
then maximal silicate or nitrate uptake is frequently shut down and there is a lag in uptake for several hours (Dortch et al. 1982, Parslow et al. 1984c, Collos 1986).

The effect of other environmental factors (in addition to substrate concentration and time discussed here) on rapid uptake has not been well studied. Rapid uptake of an ammonium analogue, methylamine, is not affected by light (Balch 1985). A decrease in temperature of the culture extended the duration of the rapid uptake $\left(V_{s}\right)$ for nitrate considerably (Raimbault 1984).

While a great deal of attention has recently been focussed on rapid nutrient uptake, mostly for a few fastgrowing diatom species, it is important to realize that not all species possess this ability. Species such as Pavlova lutheri (Burmaster \& Chisholm 1979), Scenedesmus sp. (Rhee 1978), and Chaetoceros debilis under $\mathrm{NH}_{4}^{+}$limitation (Conway et al. 1976), to name but a few, do not exhibit rapid nutrient uptake.

\section{Comparison of 3 methods of measuring nutrient uptake rates}

Method 1 (Fig. 1) was used to measure the uptake rate and the true $T=0$ concentration (the concentration determined by adding a known amount of nutrient to filtered culture effluent) was used, the uptake rates were highest at lower substrate concentrations (Fig. 3A). The reason for this observation can be seen from Fig. 2 where $V_{\mathrm{m}}$ decreases with time. In this method, the lower substrate concentrations were incubated for a shorter time than the higher substrate concentrations, because the criterion for determining incubation time was the length of time it took for approximately half the initial substrate concentration to be taken up. If the initial substrate sample is taken 2 min after the addition, then the $V$ vs $S$ curve is more hyperbolic because the rapid uptake which occurs in the first $2 \mathrm{~min}$ is omitted (Fig. 3A). But the consequence of this is shown in Fig. 3A (open circles) where it is seen that the maximal uptake rate is underestimated. Variations of Method 1 were used in earlier studies (Eppley \& Coatsworth 1968, Eppley \& Thomas 1969, Eppley et al. 1969, Carpenter \& Guillard 1971, Eppley \& Renger 1974, Underhill 1977, Serra et al. 1978, Halterman \& Toetz 1984), but the method has been used less frequently in recent years. If the time course of disappearance of the limiting nutrient from the medium is nonlinear, then this method should not be used to determine $V_{\mathrm{m}}$.

The results from Method 2 are shown in Fig. 3B. If one were using Method 2 to determine $V_{m}, 2$ min (or even shorter) incubations should be conducted. We have included longer incubations in the same figure to show clearly the decrease in $V_{\mathrm{m}}$ as incubation time increases (i.e. $V_{\mathrm{m}}^{0-5}, V_{\mathrm{m}}^{0-17}, V_{\mathrm{m}}{ }^{0-30}<V_{\mathrm{m}}^{0-2}$ ). $V_{\mathrm{m}}$ decreases rapidly as the incubation time is increased from 2 min to $30 \mathrm{~min}$ (Table 1). The rate of this decline is speciesspecific and may be due to feedback inhibition from the filling of an internal pool (Conway et al. 1976, Rhee

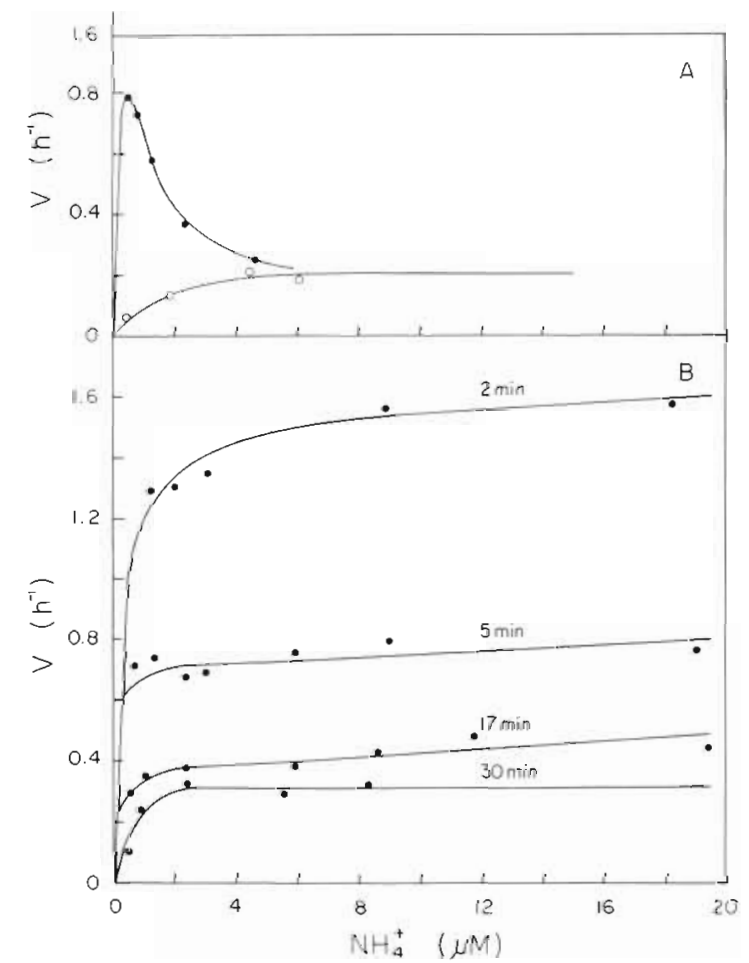

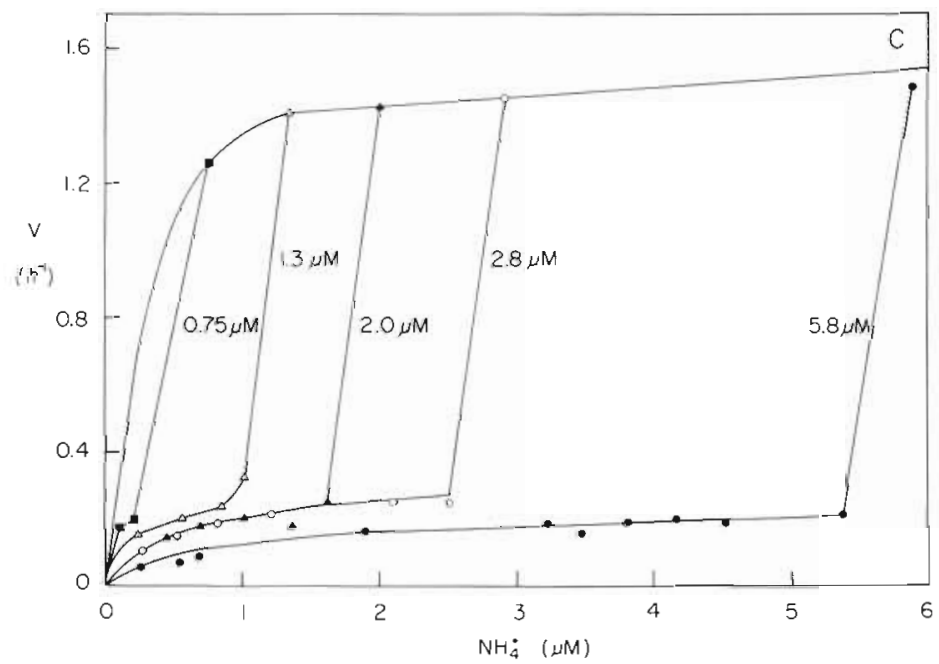

Fig. 3. Ammonium uptake rates for $\mathrm{NH}_{4}^{+}$-limited Pseudopedinella pyriformis using: (A) Method 1 - variable incubation times, with true initial substrate concentration (-) and the substrate concentration after mixing for 2 min (O); (B) Method 2 - short, constant incubation times of $2,5,17$ and 30 min; and (C) Method 3 - different sized perturbations [5.8(•), $2.8(0), 2(\Delta), 1.3(\Delta)$ and $0.75(\mathbf{}) \mu \mathrm{M} \mathrm{NH}_{4}$ ] resulting in variable past history and constant uptake intervals 
Table 1. $V_{m}$ values for ammonium for Pseudopedinella pyriformis as determined for different incubation times using Method 2 , in which incubation time is constant at all ammonium concentrations ( $n=3$ )

\begin{tabular}{|cc|}
\hline $\begin{array}{c}\text { Incubation time } \\
\text { (min) }\end{array}$ & $\begin{array}{c}V_{\mathrm{nu}} \pm 1 \mathrm{SD} \\
\left(\mathrm{h}^{-1}\right)\end{array}$ \\
\hline $0-2$ & $1.72 \pm .07$ \\
$0-5$ & $0.76 \pm .03$ \\
$0-11$ & $0.47 \pm .01$ \\
$0-17$ & $0.42 \pm .01$ \\
$0-30$ & $0.37 \pm .02$ \\
\hline
\end{tabular}

1978). Parslow et al. (1985a) have shown that feedback may occur on a scale of seconds (not minutes) but it is difficult to resolve uptake on this time scale with the AutoAnalyzer ${ }^{\text {(3) }}$. Probably the best way to estimate feedback inhlbition is to determine an inhibition constant (Rhee 1974). The $K_{S}$ values have not been reported due to the lack of data in the region of $K_{s}$ and the insensitivity of the AutoAnalyzer ${ }^{\circledR}$ at these low ammonium concentrations. If the slope, $\alpha$, of the linear portion of the rectangular hyperbola (i.e. $\alpha=V_{\mathrm{m}} / K_{\mathrm{S}}$ ) were not as steep as it is in this data set, then it is easy to see that by decreasing $V_{\mathrm{m}}, K_{S}$ would automatically decrease, even though the slope was the same. The problem of relying only on $K_{S}$ values to explain species competition at low substrate concentrations has been pointed out by Healey (1980) (i.e. a decrease in $V_{m}$ causes $K_{S}$ to decrease also, even though $\alpha$ is constant). Therefore, $\alpha$ is a much more useful and reliable parameter than $K_{S}$ to apply to nutrient competition between species because $\alpha$ is really an estimate of uptake rate at low substrate concentrations. Method 2 has been frequently used to determine $V_{\mathrm{m}}$ and $K_{S}$ (Paasche 1973, Tilman \& Kilham 1976, Rhee 1978, Burmaster \& Chisholm 1979, Goldman \& Glibert 1982 , Terry 1982). If the incubation times are short (e.g. 2 min or even shorter), then this method is the best one to measure $K_{S}$ and $V_{\mathrm{m}}$. However, for fast-growing species, feedback begins to occur on a time scale of seconds and therefore it is difficult to measure membrane transport that is completely free of feedback inhibition for these species.

For Method 3, the results of 5 different sized perturbations are given in Fig. $3 \mathrm{C}$. If one were using the perturbation technique to estimate $V_{i}$, then only one large nutrient addition would normally be made. This would give a series of uptake rates, $V^{0-T_{1}}, V^{T_{1}-T_{2}}$, $V^{T_{2}-T_{3}}$, etc. at different values of $S$. We have included 5 perturbations in Fig. $3 \mathrm{C}$ to show how the uptake parameters change with the size of the nutrient addition. $V_{m}$ appears to be relatively constant. The uptake rate drops quickly after the first few minutes of incubation to a specific uptake rate of about $0.2 \mathrm{~h}^{-1}$. This rate has been termed $V_{1}$, the internally controlled uptake rate, and it may approximate the rate of incorporation of the substrate into amino acids and other compounds of low molecular weight. If one joins the uptake rates that were measured during the first few minutes, a rectangular hyperbola is formed (Fig. $3 \mathrm{C}$ ) that is very similar to the one obtained for the $2 \mathrm{~min}$ incubations using Method 2 (Fig. 3B). On the other hand, if one omits the first 2 min of uptake of each of the 5 perturbations in Fig. $3 \mathrm{C}$, a rectangular hyperbola may be fit to the data to obtain estimates of $V_{\mathrm{i}}$ (Table 2). Parslow et al.

Table 2. $V_{\mathrm{i}}$ values for ammonium for Pseudopedinella pyriformis determined by the perturbation technique (Method 3) at different perturbation concentrations $(n=3)$

\begin{tabular}{|cc|}
\hline $\begin{array}{c}\text { Perturbation } \\
\left(\mu M N_{4}^{+}\right)\end{array}$ & $\begin{array}{c}V_{1} \pm 1 \mathrm{SD} \\
\left(\mathrm{h}^{-1}\right)\end{array}$ \\
\hline 5.8 & $0.24 \pm .02$ \\
2.8 & $0.27 \pm .02$ \\
2.0 & $0.26 \pm .03$ \\
1.3 & $0.24 \pm .04$ \\
0.75 & $0.27 \pm .04$ \\
\hline
\end{tabular}

(1985b) also found that the slope of the $V$ vs $S$ curve $(\alpha)$ was constant and independent of the exposure time to a pulse of the limiting nutrient. The perturbation technique was first used by Caperon \& Meyer (1972) and it is still frequently used with some variations in the exact procedure (Conway et al. 1976, Conway 1977. Conway \& Harrison 1977, Harrison \& Davis 1977, Burmaster \& Chisholm 1979, Turpin \& Harrison 1979, Collos 1980, 1983, 1984, Dortch et al. 1982, Lehman \& Sandgren 1982, Quarmby et al. 1982, Parslow et al. 1984 a, b, c, Raimbault 1984, Raimbault \& Mingazzini 1987). This method has the advantage of estimating $V_{\mathrm{i}}$. If a series of perturbations are conducted at different substrate concentrations and the sampling interval is short, then accurate measurements of $V_{\mathrm{m}}$ can also be obtained.

The importance of considering $V_{\mathrm{i}}$ along with $V_{\mathrm{m}}$ as a useful kinetic parameter in explaining species competition for the limiting nutrient is shown in Fig. 4. Skeletonema costatum and Chaetoceros debilis were grown in Si-limited chemostats and perturbed with $6 \mu M$ Si. Si uptake rates were determined according to Method 3. During the first $30 \mathrm{~min}, \mathrm{~S}$. costatum had a higher uptake rate than $C$. debilis; this pattern was repeated in a second experiment (Fig. 6A, B). It is apparent that $S$. costatum may outcompete $C$. debilis under conditions of silicate limitation if small pulses of silicate were added (i.e. the pulses would be taken up by the cells in less than $30 \mathrm{~min}$ ), assuming these 


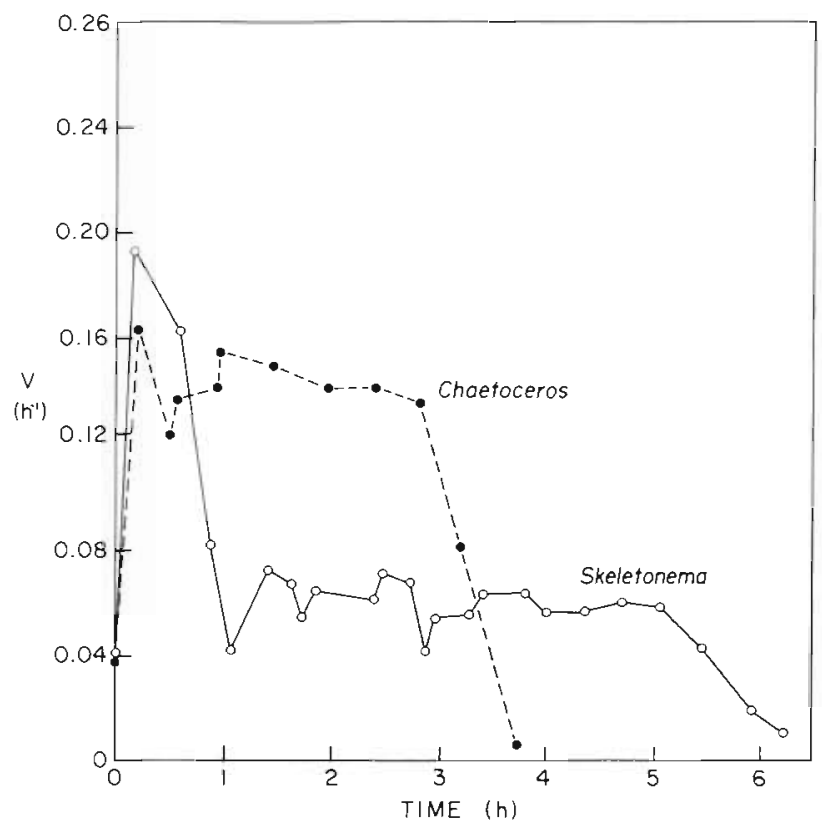

Fig. 4. Silicate uptake rates for Skeletonema costatum (o) and Chaetoceros debilis ( $\bullet$ grown in Si-limited chemostats (dilution rate $=0.3 \mathrm{~d}^{-1}$ ) and perturbed with $6 \mu \mathrm{M}$ Si. Silicate was exhausted after 3 and $5 \mathrm{~h}$ for $C$. debilis and $S$. costatum, respectively

2 species have similar Si cell quotas. However if larger pulses were added, then $C$. debilis would have competitive advantage because of its higher $V_{i}$ value and its apparent ability to assimilate silicate faster than $S$. costatum.

The perturbation technique is very amenable to the AutoAnalyzer ${ }^{(B)}$. Samples may be taken as a series of point samples every few minutes with an automated sampler (Conway et al. 1976), or by continual measurement using an in-line filter to remove the cells (Parslow et al. 1984a). A promising new technique is the loading of cells onto a filter and then running the nutrient solution past the cells (Parslow et al. 1985a). This method has the important advantage of being able to maintain the concentration of the limiting nutrient at a constant value during the perturbation.

\section{Multiple sequential additions}

These experiments are really just an extension of Method 3 to determine how cells respond to several small nutrient additions compared to one large nutrient addition in a perturbation experiment. Small $(\sim 2 \mu M)$ multiple sequential additions of ammonium to $\mathrm{NH}_{4}^{+}$limited Skeletonema costatum and Chaetoceros debilis did not affect the rate of decrease of ammonium from the medium (Fig. 5) and the uptake rate (Fig. 5 inset).
From the results shown in Fig. 2, one might expect the uptake rate to slow with time (depending on the cell density in the culture). It is interesting to note that for $S$. costatum the slope, $\alpha$, of the linear portion of the rectangular hyperbola was not affected by the repeated additions; this is less clear for C. debilis (Fig. 5 inset). This has also been clearly shown for Thalassiosira pseudonana that repeated small nutrient additions did not affect $\alpha$ (Parlsow et al. 1985b).

Larger multiple additions of silicate to Si-limited Skeletonema costatum and Chaetoceros debilis did cause the uptake rate to slow down over time (Fig. 6). The main difference for $S$. costatum was that the rapid or enhanced silicate uptake rate that occurred over the first $30 \mathrm{~min}$ during the first $\mathrm{Si}$ addition was absent during the second and third additions (Fig. 6A). The $V_{\mathrm{i}}$ portion was, on average, lower during the second and third additions. There was no change in $\alpha$ during the 3 additions (data not shown). C. debilis showed similar results to $S$. costatum but with a more pronounced effect on $V_{i}$. For the second and third additions, the $V_{\mathrm{i}}$ values continued to decrease rapidly even when silicate concentrations in the medium were not limiting or mildly limiting (e.g. 1 to $2 \mu \mathrm{MSi}$ ). Therefore, C. debilis may outcompete $S$. costatum for one large Si pulse (because of its higher $V_{1}$ value), but exposure to repeated Si pulses decreases the competitive ability of $C$. debilis ( $V_{1}$ decreases markedly). Therefore, it is impor-

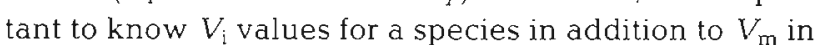
order to explain how species compete for nutrient pulses.

The initial rapid uptake of silicate by Si-starved cells has been ascribed to the Si-starvation cell synchrony effect (Sullivan 1977, Paasche 1980). A Si spike to the culture may induce rapid Si uptake and burst of valve formation. After valve formation, Si uptake slows until another set of valves are formed when the cells go through the whole cell cycle again. This Si-starvation synchrony effect may be an alternate explanation for the successively smaller $V$ values during the multiple Si additions, shown in Fig. 6A, B.

There are few examples in the literature of multiple sequential nutrient additions to phytoplankton. Lehman \& Sandgren (1982) found that 3 additions of 0.5 or 1.0 $\mathrm{MM} \mathrm{PO}_{4}$, to lake phytoplankton did not affect uptake rates. However, if 3 sequential additions of $2 \mu \mathrm{MPO}_{4}$ were added, phosphate uptake rates for the second and third additions slowed significantly (see their Fig. 7). Their results agree with our observations that several repeated small additions had no effect on the uptake kinetics, but when fewer, and larger silicate additions were made to Si-limited cultures, the silicate uptake rates of the second and third $\mathrm{Si}$ additions slowed down. Unfortunately we did not do large and small nutrient spikes for both silicate and ammonium 

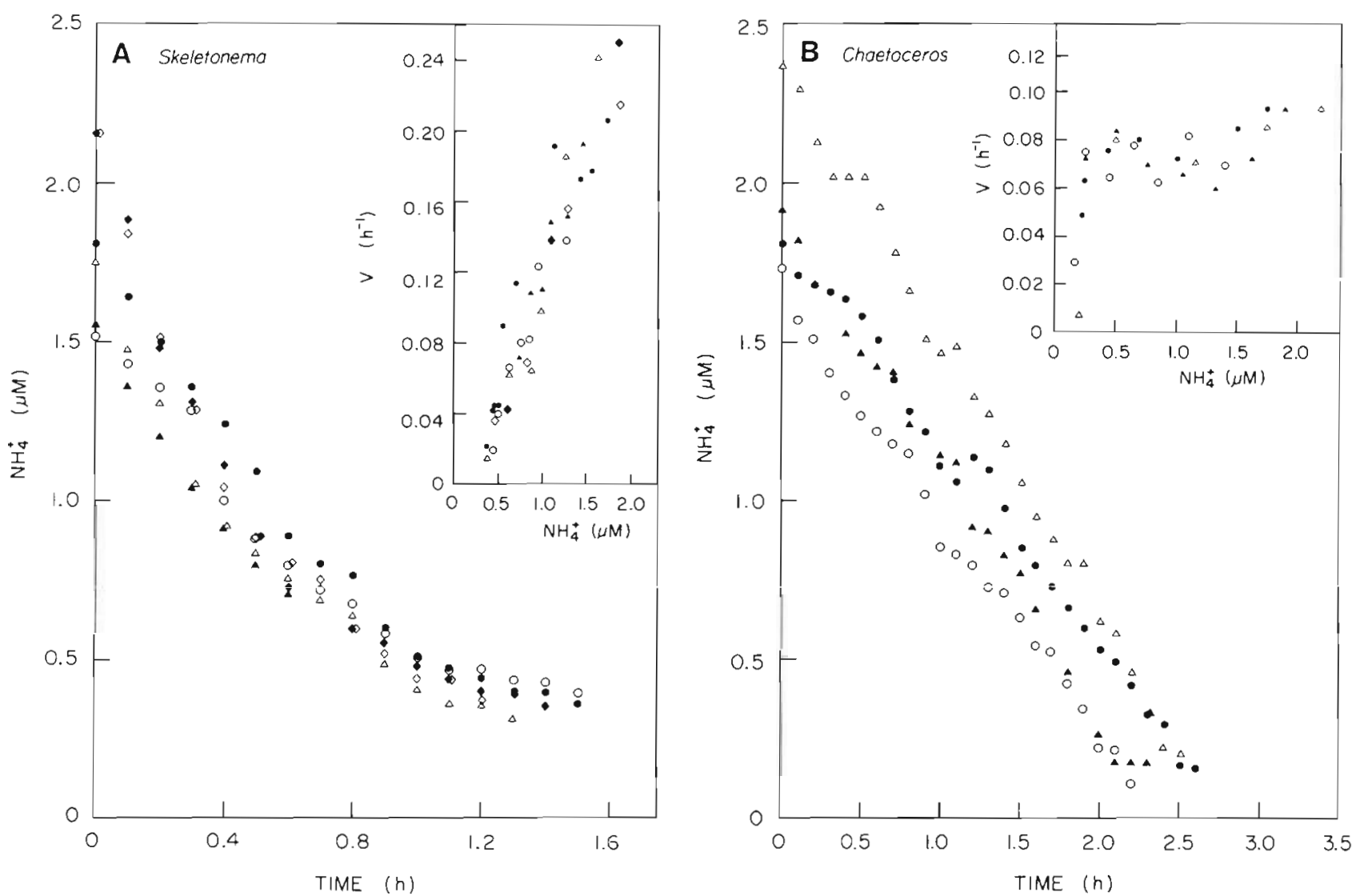

Fig. 5. Multiple or serial additions of about $2 \mu \mathrm{M}$ ammonium to $\mathrm{NH}_{4}$-limited (A) Skeletonema costatum and (B) Chaetoceros debilis and consequential disappearance of ammonium from the medium and ammonium uptake rates. Symbols indicate order of nutrient additions $(\bullet, \bullet, \Delta, \Delta, \bullet, \diamond$ represent first, second, third, fourth, fifth and sixth additions, respectively)

and therefore interpretation of our data is not as clear as Lehman \& Sandgren's data. However, one must remember that the culture density and temperature as well as the size of the nutrient addition are important because all these factors determine how long cells are exposed to elevated nutrient concentrations.

\section{Differences among nutrients in the extent of a variable $V_{\mathrm{m}}$}

Phosphate and ammonium starved or limited cells show uptake rates that are several orders of magnitude higher than their maximal growth rates $\left(\mu_{\mathrm{m}}\right)$. For example, for phosphate $V_{\mathrm{m}} / \mu_{\mathrm{m}}=5$ to 50 (Cembella et al. 1984) and for ammonium $V_{\mathrm{m}} / \mu_{\mathrm{m}}=2$ to 20 (Parslow et al. $1984 \mathrm{C}$ ). This means that for the high uptake rates of phosphate, the cells can take up enough of the nutrient in a few hours to double their cell quota. The disappearance of these 2 nutrients is generally non-linear with time and Methods 2 and 3 are recommended for determining uptake kinetic parameters. A superscript, which includes the time interval over which the maxi- mal uptake rate was determined, should be placed on the $V_{\mathrm{m}}$ term.

In order to accurately measure $V_{\mathrm{m}}$ for nutrients such as ammonium and phosphate which often show pronounced enhancement of uptake rates, very short incubation times are required. A new technique of loading cells on a filter has allowed Parslow et al. (1985a) to determine $V_{\mathrm{m}}$ over a $15 \mathrm{~s}$ interval before it begins to slow down. $V_{\mathrm{m}}$ may exhibit mild enhancement when the algae are grown in chemostats under moderate nitrate or silicate limitation (e. $\mathrm{g} . V_{\mathrm{m}} / \mu_{\mathrm{m}}=<$ ). However $24 \mathrm{~h}$ starved nitrate or silicate cells often exhibit a reduction or lag in nutrient uptake for several hours after the limiting nutrient has been added. This type of slow response is more amenable to using point sampling where the frequency of sampling can be regulated by the response of the organism. This initial lag in uptake rate is also an example of non-linear disappearance; only in this example, uptake rate speeds up with increasing incubation time, rather than slowing down as in the case of enhanced uptake rates.

A hypothetical plot of nitrate or silicate uptake rate over time is shown in Fig. $7 \mathrm{~A}_{\text {for }} \mathrm{NO}_{3}$ or Si-starved 

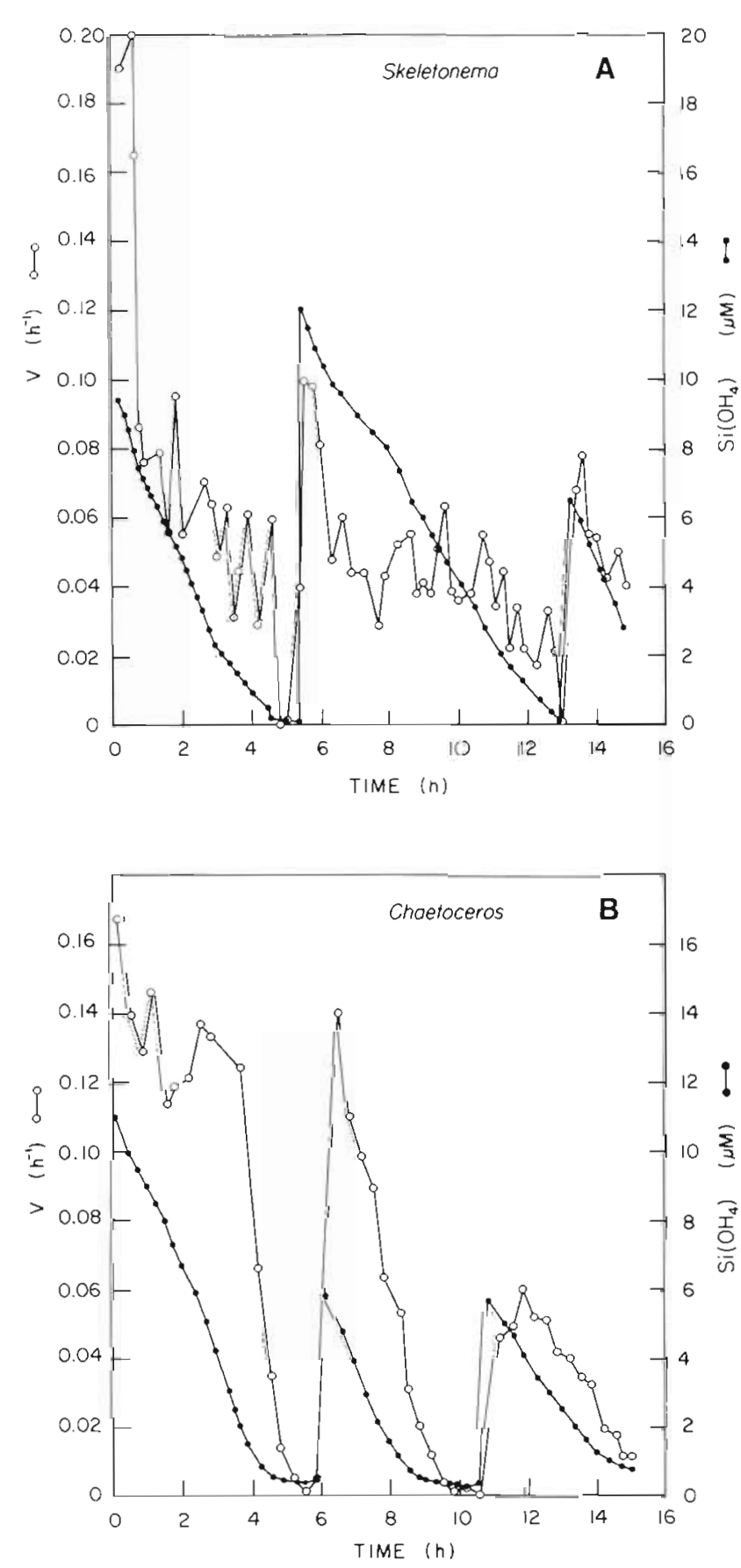

Fig. 6. Multiple sequential additions of about 6 to $10 \mu \mathrm{M}$ silicate were made to Si-limited (A) Skeletonema costatum and (B) Chaetoceros debilis, and the decrease of silicate from the medium ( $\bullet$ ) and silicate uptake rates (0) over time are shown

cells. The sequence usually is: no uptake, followed by an inducement period, and then maximal uptake rate several hours after the nitrate or silicate addition. The results of $V$ vs $S$ plots for the 3 methods are shown in Fig. $7 \mathrm{~B}$ to $\mathrm{D}$ and raise the obvious question, when do
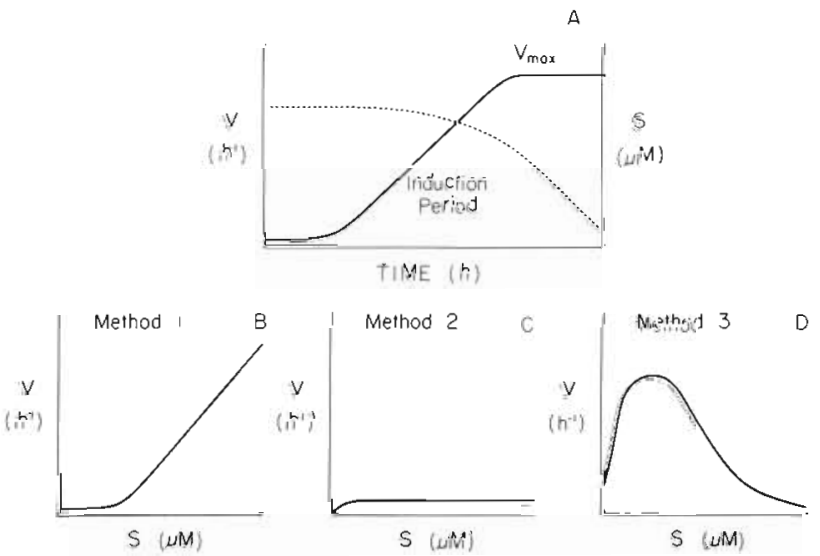

Fig. 7. (A) Hypothetical plot of nitrate or silicate uptake rates (solid line) over time after the addition of nitrate or silicate (dashed line) to nitrate- or silicate-starved cells. ( $B$ to $D$ ) Hypothetical plots of uptake rate versus nitrate or silicate for Methods 1,2 and 3, respectively

you measure nitrate and silicate uptake rates in order to measure maximal rates?

\section{Ecological implications}

Enhanced uptake of the limiting nutrient has also been observed in the field. Natural phytoplankton assemblages off the east coast of the USA showed a 5 min rate that was 4 times higher than the 2 h uptake rate (Glibert \& Goldman 1981). For Chesapeake Bay phytoplankton, where relatively severe nitrogen stress was demonstrated, Glibert \& McCarthy (1984) found that the uptake rate of nitrate was only mildly enhanced, whereas ammonium uptake rates were enhanced 5- to 10 -fold relative to longer incubations. Other examples of significant enhancement in ammonium uptake rates were in the surf zone along the northwest coast of USA (Collos \& Lewin 1974), an estuary in North Carolina (Fisher et al. 1981), Chesapeake Bay (Wheeler et al. 1982), in the Canadian Arctic (Harrison 1983) and in a freshwater lake (Priscu 1987). Rapid ammonium uptake has also been observed in intertidal seaweeds grown under field conditions (Thomas \& Harrison 1987). Results from laboratory experiments indicate that it may not be possible to do short enough incubations with ${ }^{15} \mathrm{~N}$ to obtain $V_{\mathrm{m}}$ in the field. There are only a few examples of enhanced phosphate uptake in marine waters (Lemasson et al. 1980, Berman 1983), but there are numerous examples for lakes (Lean \& White 1983) and cultures of freshwater phytoplankton (Suttle \& Harrison 1988) where short-term uptake of phosphate may be 10 to 100 times higher than maximum growth rates. 
A lag in nitrate uptake rate has been observed in the field (Maclsaac \& Dugdale 1969). If time courses are not run for ${ }^{15} \mathrm{~N}$ field experiments, then it is difficult to interpret nitrate uptake rates because rates may increase with increasing incubation time (Fig. 7 A).

It is clear from our results and discussions that all limiting nutrients do not show a similar pattern of response to the addition of the limiting nutrient. Dortch et al. (1982) have made a similar observation and they have divided the nutrients into 2 groups, the recycled nutrients, ammonium and phosphate, and the advected/upwelled nutrients, nitrate and silicate. The recycled nutrients show an immediate uptake response to nutrient additions while the advected nutrients may exhibit a lag in uptake rate especially if the cells have previously been nutrient-starved. It is important to realize that the maximal uptake of nutrients is frequently not constant under nutrient-limited conditions, and that a time course incubation must be run to determine the complete pattern of response of the limiting nutrient to a nutrient addition.

\section{Recommendations}

(1) A time course of the disappearance of the limiting nutrient must be run to determine if it is linear or nonlinear, particularly if nutrient limitation is suspected. In the field it may not be possible to do short enough incubations (e. g. 2 min or less) with ${ }^{15} \mathrm{~N}$ to obtain $V_{\mathrm{m}}$ for ammonium, under $\mathrm{N}$-limited conditions.

(2) It is important to realize that non-linear disappearance of the limiting nutrient can occur in 2 patterns, as an enhancement of uptake or as a lag in uptake. If the disappearance is non-linear then: (a) $V_{m}$ must be determined using Method 2 (very short incubation times at a range of substrate concentrations). The incubation time interval should be denoted as a superscript on the $V_{\mathrm{m}}$ term; (b) the initial substrate concentration at $T=0$ should be determined by adding a known volume of substrate to culture filtrate or to a dilute culture; (c) Method 3 (a perturbation technique) should be used to determine $V_{1}$. Consequently for nonlinear disappearance, 3 kinetic parameters should be reported, $V_{m}{ }^{0-2}, V_{1}$ and $\alpha_{i}$ (d) when nitrate- or silicatestarved cells exhibit a lag in nitrate or silicate uptake rate, a series of $V_{m}$ values should be reported (e. $\mathrm{g}$. $V_{\mathrm{m}}{ }^{0-5}, V_{\mathrm{m}}{ }^{\mathrm{s}-15}, V_{\mathrm{m}}{ }^{15-60}$ ) in order to document the time series increase in $V_{\mathrm{m}}$

Acknowledgements. This research was supported by a grant from the Natural Sciences and Engineering Research Council of Canada. Part of this research was conducted at the Radiological and Environmental Research Division, Argonne National Laboratory, Argonne, Illinois, USA. Technical assistance by Susan Williams is gratefully acknowledged.
Thorough reviews by Drs Y Collos, Q. Dortch, E. Paasche and in particular, E. Laws, greatly improved this paper. Comments on a draft of this manuscript by W. Cochlan, P. A. Thompson, $M$. Lavasseur and A. Waite were very valuable.

\section{LITERATURE CITED}

Balch, W. M. (1985). Lack of an effect of light on methylamine uptake by phytoplankton. Limnol. Oceanogr. 30: 665-674

Berman, T. (1983). Phosphorus uptake by microplankton in estuarine and coastal shelf waters near Sapelo Island, Georgia, U.S.A. Estuaries 6: 160-166

Burmaster, D. E., Chisholm, S. W. (1979). A comparison of two methods for measuring phosphate uptake by Monochrysis luthen Droop grown in continuous culture. J. exp. mar Biol. Ecol. 39: 187-202

Button, D. K. (1985). Kinetics of nutrient-limited transport and microbial growth. Microbiol. Rev. 49: 270-297

Caperon, J., Meyer, J. (1972). Nitrogen-limited growth of marine phytoplankton. Part II. Uptake kinetics and their role in nutrient-limited growth of phytoplankton. Deep Sea Res. 19: 619-632

Carpenter, E. J., Guillard, R. R. L. (1971). Intraspecific differences in nitrate half-saturation constants for three species of marine phytoplankton. Ecology 52: 183-185

Cembella, A. D., Antia, N. J., Harrison, P. J. (1984). The utilization of inorganic and organic phosphorus compounds as nutrients by eukaryotic microalgae: a multidisciplinary perspective: Part I. CRC Crit. Rev. Microbiol. 10: 317-391

Cleland, W (1967). The statistical analysis of enzyme kinetic data. Adv. Enzymol. 29: 1-32

Collos, Y (1980). Transient situations in nitrate assimilation by marine diatoms. I. Changes in uptake parameters during nitrogen starvation. Limnol. Oceanogr. 25: 1075-1081

Collos, Y. (1983). Transient situations in nitrate assimilation by marine diatoms. IV Non-linear phenomena and the estimation of the maximum uptake rate. J. Plankton Res. 5: $677-691$

Collos, Y. (1984). Transient situations in nitrate assimilation by marine diatoms. $V$ Interspecific variability in biomass and uptake during nitrogen starvation and resupply. Mar. Ecol. Prog. Ser. 17: 25-31

Collos, Y (1986). Time-lag algal growth dynamics: biological constraints on primary production in aquatic environments. Mar. Ecol. Prog. Ser, 33: 193-206

Collos, Y., Lewin, J. (1974). Blooms of surf-zone diatoms along the coast of the Olympic Peninsula, Washington. IV Nitrate reductase activity in natural populations and laboratory cultures of Chaetoceros armatum and Asterionella socialis. Mar. Biol. 25: 213-221

Conway, H. L. (1977). Interactions of inorganic nitrogen in the uptake and assimilation by marine phytoplankton. Mar. Biol. 39: 221-232

Conway, H. L., Harrison, P. J. (1977). Marine diatoms grown in chemostats under silicate or ammonium limitation. IV Transient response of Chaetoceros debilis, Skeletonema costatum, and Thalassiosira gravida to a single addition of the limiting nutrient. Mar Biol. 43: 33-43

Conway, H. L., Harrison, P. J., Davis, C. O. (1976). Marine diatoms grown in chemostats under silicate or ammonium limitation. II. Transient response of Skeletonema costatum to a single addition of the limiting nutrient. Mar. Biol. 35: 187-199

Davis, C. O., Harrison, P. J., Dugale, R. C. (1973). Continuous 
culture of marine diatoms under silicate limitation. I. Synchronized life cycle of Skeletonema costatum. J. Phycol. 9: 175-180

Dortch, Q., Clayton, J. R. Jr, Thoressen, S. S., Bressler, S. L., Ahmed, S. I. (1982). Response of marine phytoplankton to nitrogen deficiency: decreased nitrate uptake vs enhanced ammonium uptake. Mar. Biol. 70: 13-19

Dugdale, R. C. (1967). Nutrient limitation in the sea: dynamics, identification, and significance. Limnol. Oceanogr 12: 685-695

Dugdale, R. C., Goering, J. J. (1967). Uptake of new and regenerated forms of nitrogen in primary productivity. Limnol. Oceanogr 12: 196-206

Eppley, R. W., Coatsworth, J. L. (1968). Nitrate and nitrite uptake by Ditylum brightwellii: kinetics and mechanisms. J. Phycol. 4: 151-156

Eppley, R. W., Renger, E. H. (1974). Nitrogen assimilation of an oceanic diatom in nitrogen-limited culture in a chemostat. J. Phycol. 10: 15-23

Eppley, R. W., Rogers, J. N., McCarthy, J. J. (1969). Half saturation 'constants' for uptake of nitrate and ammonium by marine phytoplankton. Limnol. Oceanogr 14: 912-920

Eppley, R. W., Thomas, W. J. (1969). Comparison of halfsaturation constants for growth and nitrate uptake of marine phytoplankton. J. Phycol. 5: 375-379

Fisher, T. R., Carlson, P. R., Barber, R. T (1981). Some problems in the interpretation of ammonium uptake. Mar. Biol. Lett. 2: 33-44

Glibert, P. M., Goldman, J. C. (1981). Rapid ammonium uptake by marine phytoplankton. Mar. Biol. Lett. 2: 25-31

Glibert, P. M., McCarthy, J. J. (1984). Uptake and assimilation of ammonium and nitrate by phytoplankton: indices of nutritional status for natural assemblages. J. Plankton Res. 6: $677-697$

Goldman, J. C., Glibert, P. M. (1982). Comparative rapid ammonium uptake by four species of marine phytoplankton. Limnol. Oceanogr. 27: 814-827

Goldman, J. C., Glibert, P. M. (1983). Kinetics of inorganic nitrogen uptake by phytoplankton, In: Carpenter, E. J., Capone, D. G. (eds.) Nitrogen in the marine environment. Academic Press, New York, p. 233-274

Goldman, J. C., Taylor, C. D., Glibert, P. M. (1981). Nonlinear time-course uptake of carbon and ammonium by marine phytoplankton. Mar Ecol. Prog. Ser 6: 137-148

Guillard, R. R. L., Ryther, J. H. (1962). Studies of marine plankton diatoms. I. Cyclotella nana (Hustedt) and Detonula confervacea (Cleve). Gran. Can. J. Microbiol. 8: $229-239$

Halterman, S. G., Toetz, D. W (1984). Kinetics of nitrate uptake by freshwater algae. Hydrobiol. 114: 209-214

Harrison, P. J., Conway, J. L., Holmes, R. W., Davis, C. O. (1977). Marine diatoms grown in chemostats under silicate or ammonium limitation. III. Cellular chemical composition and morphology of Chaetoceros debilis, Skeletonema costatum, and Thalassiosira gravida. Mar. Biol. 43: 19-31

Harrison, P. J., Davis, C. O. (1977). Use of the perturbation technique to measure nutrient uptake rates of natural phytoplankton populations. Deep Sea Res. 24: 247-255

Harrison, W. G.(1983). The time-course of uptake of inorganic and organic nitrogen compounds by phytoplankton from the Eastern Canadian Arctic: a comparison with temperate and tropical populations. Limnol. Oceanogr 28: 1231-1237

Healey, F. P. (1980). Slope of the Monod equation as an indicator of advantage in nutrient competition. Microbial. Ecol. 5: 281-286

Lean, D. R. S., White, E. (1983). Chemical and radiotracer measurements of phosphorus uptake by lake plankton. Can. J. Fish. Aquat. Sci. 40: 147-155

Lehman, J. T., Sandgren, C. D. (1982). Phosphorus dynamics of the procaryotic nannoplankton in a Michigan lake. Limnol. Oceanogr. 27. 828-838

Lemasson, L., Pagès, J., Cremoux, J.-L. (1980). Inorganic phosphate uptake in a brakish tropical lagoon. Estuar. coast. mar Sci. 11: 547-561

Maclsaac, J. J., Dugdale, R. C. (1969). The kinetics of nitrate and ammonia uptake by natural populations of marine phytoplankton. Deep Sea Res. 16: 45--57

McCarthy, J. J., Goldman, J. C. (1979). Nitrogenous nutrition of marine phytoplankton in nutrient-depleted waters. Science 203: 670-672

Nelson, D. M., Goering, J. J. (1977), A stable isotope tracer method to measure silicic acid uptake by marine phytoplankton. Analyt. Biochem. 78: 139-147

Paasche, E. (1973). Silicon and the ecology of marine plankton diatoms. II. Silicate-uptake kinetics in five diatom species. Mar. Biol. 19: 117-126

Paasche, E. (1980). Silicon. In: Morris, I. (ed.) The physiological ecology of phytoplankton. Blackwell Sci. Publ., Oxford, p. $259-284$

Parslow, J. S., Harrison, P. J., Thompson, P. A. (1984a). Use of a self-cleaning in-line filter to continuously monitor phytoplankton nutrient uptake rates. Can. J. Fish. Aquat. Sci. 41: $540-544$

Parslow, J. S., Harrison, P. J., Thompson, P. A. (1984b). Development of rapid ammonium uptake during starvation of batch and chemostat cultures of the marine diatom Thalassiosira pseudonana. Mar. Biol. 83: 43-50

Parslow, J. S., Harrison, P. J., Thompson, P. A. (1984c). Saturated uptake kinetics: transient response of the marine diatom Thalassiosira pseudonana to ammonium, nitrate, silicate or phosphate stavation. Mar. Biol. 83: 51-59

Parslow, J. S., Harrison, P. J., Thompson, P. A. (1985a). Ammonium uptake by phytoplankton cells on a filter: a new high-resolution technique. Mar Ecol. Prog. Ser. 25: $121-129$

Parslow, J. S., Harrison, P. J., Thompson, P. A. (1985b). Interpreting rapid changes in uptake kinetics in the marine diatom Thalassiosira pseudonana (Hustedt). J. exp. mar. Biol. Ecol. 91: 53-64

Priscu, J. C. (1987). Time-course of inorganic nitrogen uptake and incorporation by natural populations of freshwater phytoplankton. Freshwat. Biol. 17: 331-339

Quarmby, L. M., Turpin, D. H., Harrison, P. J. (1982). Physiological responses of two marine diatoms to pulsed additions of ammonium. J. exp mar. Biol. Ecol. 63: 173-181

Raimbault, P. (1984). Influence of temperature on the transient response in nitrate uptake and reduction by four marine diatoms. J. exp. mar. Biol. Ecol. 84: 37-53

Raimbault, P. Mingazzini, M. (1987). Diurnal variations of intracellular nitrate storage by marine diatoms: effects of nutritional state. J. exp. mar. Biol. Ecol. 112: 217-232

Rhee, G. Y (1974). Phosphate uptake under nitrate limitation by Scenedesmus sp. and its ecological implications. J. Phycol. 10: 470-475

Rhee, G. Y (1978). Effects of $\mathrm{N}: \mathrm{P}$ atomic ratios and nitrate limitation on algal growth, cell composition, and nitrate uptake. Limnol. Oceanogr 23: 10-23

Serra, J. L., Llama, M. J., Cadenas, E. (1978). Nitrate utilization by the diatom Skeletonema costatum. Il. Regulation of nitrate uptake. Plant Physiol. 62: 991-994

Sullivan, C. (1977). Diatom mineralization of silicic acid. II. Regulation of $\mathrm{Si}(\mathrm{OH})_{4}$ transport rates during the cell cycle of Navicula pelliculosa. J. Phycol. 13: 86-91 
Suttle, C. A., Harrison, P. J. (1988). Rapid ammonium uptake by freshwater phytoplankton. J. Phycol. 24: 13-16

Syrett, P. J. (1956). The assimilation of ammonia and nitrate by nitrogen-starved cells of Chlorella vulgaris. II. The assimilation of large quantities of nitrogen. Physiol. Pl. 9: $19-27$

Terry, K. L. (1982). Nitrate and phosphate uptake interactions in a marine prymnesiophyte. J. Phycol. 18: 79-86

Thomas, T. E., Harrison, P. J. (1987). Rapid ammonium uptake and nitrogen interactions in five intertidal seaweeds grown under field conditions. J. exp. mar. Biol. Ecol. 107: 1-8

Tilman, D. (1977). Resource competition between planktonic algae: an experimental and theoretical approach. Ecology 58: $338-348$

This article was presented by Professor E. Paasche,

Oslo, Norway
Tilman, D., Kilham, S. S. (1976). Phosphate and silicate growth and uptake kinetics of the diatoms Asterionella formosa and Cyclotella meneghiniana in batch and semicontinuous culture. J. Phycol. 12: 375-383

Turpin, D. H. Harrison, P. J. (1979). Limiting nutrient patchiness and its role in phytoplankton ecology. J. exp. mar. Biol. Ecol. 39: 151-156

Underhill, P. A. (1977). Nitrate uptake kinetics and clonal variability in the neritic diatom Biddulphic aurita. J. Phycol. 13: 170-176

Wheeler, P. A., Glibert, P. M., McCarthy, J. J. (1982). Ammonium uptake and incorporation by Chesapeake Bay phytoplankton: short-term uptake kinetics. Limnol. Oceanogr. 27: 1113-1128

Manuscript received: December 17, 1987

Revised version accepted: December 23, 1988 